Pacific Journal of Mathematics

PROPERTIES OF FIXED POINT SETS ON DENDRITES 


\title{
PROPERTIES OF FIXED POINT SETS ON DENDRITES
}

\section{HELGA SCHIRMER}

\begin{abstract}
Every nonempty closed subset of a dendrite can be the fixed point set of a self-map, but in general it cannot be the fixed point set of a map with special properties. Necessary conditions found here for the fixed point sets of homeomorphisms and monotone surjections of dendrites are mainly concerned with the order of the possible fixed points, and extend earlier results by G. E. Schweigert and L. E. Ward, Jr.
\end{abstract}

1. Introduction. It was proved in [3,4] that every closed, nonempty subset of the $n$-ball $B^{n}$ can be the fixed point set of a self-map of $B^{n}$, but that not all such subsets can be the fixed point set of a homeomorphism of $B^{n}$. We investigate in this paper related questions for dendrites. The first result (Theorem 3.1) shows that again every closed nonempty subset can be the fixed point set of a self-map of a dendrite.

It is already known that not every closed nonempty subset $A$ of a dendrite $D$ can be the fixed point set of a homeomorphism of $D$, or even of a monotone surjection of $D$. Results for homeomorphisms by G. E. Schweigert [5] and generalizations for monotone maps by L. E. Ward, Jr. [7] show that $A$ cannot consist of one end point of $D$ :

THEOREM 1.1. (Schweigert and Ward). Let $f: D \rightarrow D$ be a monotone surjestion of a dendrite $D$ which leaves one end point $e$ of $D$ fixed. Then there exists at least one fixed point distinct from $e$.

We extend this theorem in several ways. In $\$ 4$ we prove more details about the order (see [8, p. 48]) of the possible fixed points if the fixed point set consists of only finitely many points. The theorem by Schweigert and Ward states that the fixed point set of a monotone surjection cannot consist of one end point, i.e., of one point of order one. We show in Theorem 4.1 that it also cannot consist of two points of order two, and in the case of a homeomorphism it cannot consist of three points of order three. But it can consist of $n$ points of order $n$ for all $n>3$. We further strengthen Theorem 1.1 by proving a restriction on the fixed point different from $e$ : if $f$ is a homeomorphism, then it can be chosen of an order $\neq 2$ (Theorem 4.5). This is no longer true for monotone surjections.

The work by Schweigert and Ward is concerned with fixed point 
sets containing one end point. In $\S 5$ we investigate fixed point sets which contain almost all of the end points, and show that they must contain also all points of a sufficiently high order (Theorem 5.1). In particular we can conclude that if a monotone surjection leaves all but one of the end points fixed, then it leaves in fact all points of order $\neq 2$ fixed (Corollary 5.5).

In $\S 4$ we saw that a distinction exists between fixed point sets of homeomorphisms and of monotone surjections. In the final paragraph (§6) we show that such a distinction no longer holds for finite dendrites, i.e., that a subset of a finite dendrite can be the fixed point set of a homeomorphism if and only if it can be the fixed point set of a monotone surjection (Theorem 6.1). The same is true for open maps of finite dendrites, but nothing is known so far about fixed point sets of open maps of arbitrary dendrites.

Ward actually proved Theorem 1.1 not only for dendrites, but more generally for trees, i.e., he did not assume that the space has a metric. It is likely that most or all of the results of this paper can be extended to trees. The metric of the dendrite is used crucially in the proof of Theorem 3.1, and it is also used implicitly in the parts of the paper concerning the order of a point as this concept was developed in [8] for the metric case.

2. Dendrites. A dendrite $D$ is a metric continuum (i.e., compact connected Hausdorff space) in which every pair of distinct points is separated by a third. We use the partial order structure of dendrites which was developed by Ward [6,7]. Take an arbitrary point $r \in D$ as root, and define a partial order $\leqq$ on $D$ by $x \leqq y$ if $x=r, x$ separates $r$ and $y$, or $x=y$. Then $r \leqq x$ for every $x \in D$. Define

$$
\begin{aligned}
L(\alpha) & =\{y \in D \mid y \leqq a\}, \\
M(\alpha) & =\{y \in D \mid a \leqq y\}
\end{aligned}
$$

The sets $L(a)$ and $M(a)$ are closed in $D$. Let $[a, b]=M(a) \cap L(b)$; it is a nonempty closed chain (i.e., it is linearly ordered) if $a<b$. Let $(a, b)$ be the interior of $[a, b]$. A point $m$ is called a maximum of a subset $A$ of $D$, written $\max A$, if $m \nless x$ for each $x \in A$. It is shown in [6, Theorem 1] that every nonempty closed subset of $D$ has a maximum.

We also need in the following some results about dendrites, in particular about the order of points and about arcwise connectedness, which can be found in [8]. Frequently we use the next lemma which 
characterizes the order $o(a)$ of $a$ point a $[8, \mathrm{p} .48]$ in the case where it is finite.

LEMma 2.1. Let $a$ be a point of a dendrite $D$. If either the order $o(a)$ or the number of the components of $D \backslash\{a\}$ is finite, then these two numbers are equal $[8, \mathrm{p} .88]$.

$a \in D$ is called an end point if $o(\alpha)=1$, a cut point if $o(a) \geqq 2$, and a branch point if $o(a) \geqq 3$.

LEMmA 2.2. Every maximum of $D$ is an end point, and every end point is either a maximum or a root.

Proof. Let $m$ be a maximum of $D$. If $m$ is not an end point, then it is a cut point [8, p. 88], and therefore $m$ separates $D$ into two disjoint separated sets $A$ and $B[8, \mathrm{p} .42]$. Choose $A$ and $B$ so that the root $r$ is in $A$, and take any $y \in B$. Then $m$ separates $r$ and $y$, i.e., $m<y$. But this is impossible if $m$ is a maximum. Hence $m$ is an end point. Let now $e$ be an end point with $e \neq r$. As $e$ is not a cut point, the set $D \backslash\{e\}$ is connected, and $e$ cannot separate any two points of $D \backslash\{e\}$. So $e<x$ is not possible for any $x \in D$, and hence $e$ is a maximum of $D$.

It follows from [6, Theorem 5] that $M(x)$ is connected for all $x \in D$, and therefore $M(x)$ is a subdendrite with root $x[8, \mathrm{p} .89]$. The space $D$, and hence $M(x)$, are not only connected, but they are also arcwise connected, and the arc between any two of their points is unique [8, p. 89]. We write arc $a b$ for the unique are from $a$ to $b$ if $a, b \in D$.

Lemma 2.3. If $b_{1}, b_{2} \in D$ and $m=\max \left[L\left(b_{1}\right) \cap L\left(b_{2}\right)\right]$, then arc $b_{1} b_{2}=\left[m b_{1}\right] \cup\left[m b_{2}\right]$.

Proof. The sets $\left[m b_{i}\right]=M(m) \cap L\left(b_{1}\right)$, where $i=1,2$, are connected chains and hence arcs [7, Theorem 1; 6, Theorems 4 and 6; 8, p. 36]. As $\left[m b_{1}\right]$ and $\left[m b_{2}\right]$ have exactly one point in common, $\left[m b_{1}\right] \cup\left[m b_{2}\right]$ is an arc, and hence it is the unique arc $b_{1} b_{2}$.

An immediate consequence of Lemma 2.3 is

Lemma 2.4. If the connected subset $A$ of $D$ contains the points $b_{1}$ and $b_{2}$, then it also contains $\max \left[L\left(b_{1}\right) \cap L\left(b_{2}\right)\right]$.

We finally state a lemma concerning homeomorphisms and monotone 
maps (i.e., maps where $f^{-1}(y)$ is connected for all points of the range of $f$ ) which is crucial in most of the following work. Its proof can be found in [6, Lemma 13 and p. 156].

Lemma 2.5. If $f: D \rightarrow D$ is a monotone surjestive self-map of a dendrite $D$, then it is isotone (i.e., $x<y$ implies $f(x) \leqq f(y)$ ). If $f: D \rightarrow D$ is a homeomorphism, then it is strictly isotone (i.e., $x<y$ implies $f(x)<f(y)$ ).

From now on all monotone surjections are assumed to be continuous.

3. Fixed point sets of arbitrary maps on dendrites. We show in this paragraph that any closed nonempty subset can be the fixed point set of a self-map of a dendrite.

THEOREM 3.1. Let $A$ be an arbitrary closed nonempty subset of a dendrite $D$. Then there exists a map $f: D \rightarrow D$ with $A$ as its fixed point set.

Proof. Give $D$ the convex metric $d$ (see $[1,2]$ ). As $D$ is acyclic and complete, it follows that for every $x, y \in D$ the point

$$
z=t x+(1-t) y \quad(0 \leqq t \leqq 1)
$$

is a unique point of $D$. As $D$ is compact, it is bounded, hence the diameter diam $(D)$ is finite. Select a point $a \in A$, and define

$$
f(x)=\frac{d(x, A)}{\operatorname{diam}(D)} a+\left[1-\frac{d(x, A)}{\operatorname{diam}(D)}\right] x \text { for every } x \in D .
$$

Then $f$ is the desired map.

Note that the result is not true any longer if we ask in addition that $f$ is surjective. It is e.g., not possible to construct a map from the unit interval onto itself such that its fixed point set consists of one end point of the interval.

4. Nonexistence of some finite fixed point sets. Theorem 1.1 by Schweigert and Ward shows that the fixed point set of a monotone surjection on a dendrite cannot consist of one point of order one. We investigate in this paragraph the existence of fixed point sets on dendrites consisting of $n$ points of order $n$, for arbitrary positive integers $n$. The main result is stated in the following theorem.

THEOREM 4.1. Let $f: D \rightarrow D$ be a surjective self-map of a dendrite. 
(i) If $f$ is monotone, then the fixed point set of $f$ cannot consist of $n$ points of order $n$ for $n=1$ or $n=2$.

(ii) If $f$ is a homeomorphism, then the fixed point set of $f$ cannot consist of $n$ points of order $n$ for $n=1, n=2$ or $n=3$.

The proof of Theorem 4.1 is lengthy and will be accomplished in several parts. The next lemma is used in the proof of part (i) of Theorem 4.1 and in the proof of Theorem 4.5 below.

LEMMA 4.2. If $a$ is a point of order two in $D$ and different from the root, then it is a point of order one in the subdendrite $M(\alpha)$.

Proof. As $o(a)=2$ in $D$, we can assume that $D \backslash\{a\}=K_{1} \cup K_{2}$, where $K_{1}$ and $K_{2}$ are the two components of $D \backslash\{a\}$ and the root $r \in K_{1}$. As $K_{1}$ is arcwise connected, we have

$$
\begin{aligned}
K_{1} & =\{x \mid a \notin \operatorname{arc} r x\} \\
& =\{x \mid a \notin[r x]\}=D \backslash M(\alpha) .
\end{aligned}
$$

Hence $K_{2}=M(a) \backslash\{a\}$, so that $M(a) \backslash\{a\}$ is connected and $o(a)=1$ in $M(a)$.

Proof of part (i) of Theorem 4.1. Because of Theorem 1.1 we only have to prove the nonexistence of a fixed point set consisting of two points of order two.

Let $f: D \rightarrow D$ be a monotone surjection which has two fixed points of order two. Take one as root $r$, and let $a$ be the other fixed point. As $\mathrm{f}$ is isotone (Lemma 2.5), we have $f(M(\alpha)) \subseteq M(\alpha)$. The restriction $f \mid M(a): M(a) \rightarrow M(a)$ is monotone, as for any $y \in f(M(a))$ the counterimage $f^{-1}(y)$ is connected in $D$ and hence (see [8, p. 88]) $f^{-1}(y) \cap M(a)$ is connected in $M(a)$. If $f \mid M(a): M(a) \rightarrow M(a)$ is onto, then it follows from Theorem 1.1 and Lemma 4.2 that $f$ has a second fixed point on $M(\alpha)$, and part (i) of Theorem 4.1 is proved.

Assume now that $f(M(a)) \neq M(a)$, and choose $q \in M(a) \backslash f(M(a))$. As $f$ is surjective, there exists $p \in D \backslash M(a)$ with $f(p)=q$, and because $f$ is isotone, we have $f([r a])=[r a]$, so that in fact $p \in D \backslash\{M(a) \cup[r a]\}$. Let $m=\max [L(p) \cap L(q)]$. Then $r \leqq m<a$ and hence $r \leqq f(m) \leqq a$. But in fact $f(m)=\alpha$ : as $f([m p])=[f(m) q]$ and $a \in[f(m) q]$, there exists an $x \in[m p]$ with $f(x)=a$. But we also have $f(a)=a$, so that by Lemma 2.4 the connected set $f^{-1}(a)$ must contain

$$
\max [L(x) \cap L(a)]=m \text {, i.e., } f(m)=a \text {. }
$$


So we see that if $f$ has no other fixed points but $r$ and $a$, then there exists $m \in$ arc $r a \backslash\{a\}$ with $f(m)=a$. If we take $a$ instead of $r$ as root, then an analogous argument shows: if $f$ has no other fixed points but $r$ and $a$, then there exists $n \in \operatorname{arc} r a \backslash\{r\}$ with $f(n)=r$. But as $f(\operatorname{arc} r a)=$ arc $r a$, the existence of $m$ and $n$ implies the existence of a fixed point on are $r a$ different from $r$ and $a$. Hence $f$ must have a fixed point different from $r$ and $a$, and part (i) of Theorem 4.1 follows.

We now set out to prove part (ii) of Theorem 4.1. This is done with the help of the next two lemmas. The first is stated in much more generality than is needed here for the sake of its use in the proof of Theorem 5.1 below. We say that $f: D \rightarrow D$ permutes the set of $n$ points $\left\{b_{i} \mid i=1,2, \cdots, n\right\}$ of $D$ if it transforms the set $\left\{b_{i}\right\}$ bijectively onto itself; the identity transformation of the $b_{i}$ is included as a possibility.

Lemma 4.3. Assume that the monotone surjection $f: D \rightarrow D$ leaves the root of $D$ fixed and that it permutes the set of points

$$
\left\{b_{i} \mid i=1,2, \cdots, n\right\},
$$

where $n \geqq 2$. Then

$$
m=\max \left[\bigcap_{i=1}^{n} L\left(b_{i}\right)\right]
$$

is a fixed point of $f$.

Proof. Let $r$ be the root of $D$. As $r \leqq m \leqq b_{i}$, the fact that $f$ is isotone (see Lemma 2.5) implies

$$
r \leqq f(m) \leqq f\left(b_{i}\right)=b_{k}(i, k=1,2, \cdots, n)
$$

and hence $f(m) \leqq m$. But $f\left(\left[r b_{i}\right]\right)=\left[r b_{k}\right]$, so that there exists for $i=1,2, \cdots, n$ an $x_{i}$ with $r \leqq m \leqq x_{i} \leqq b_{i}$ and $f\left(x_{i}\right)=m$. Therefore the connected set $f^{-1}(m)$ contains all $x_{i}$, and as

$$
\max \left[\bigcap_{i=1}^{n} L\left(x_{i}\right)\right]=\max \left\{L\left[\max \bigcap_{i=1}^{n-1} L\left(x_{i}\right)\right] \cap L\left(x_{n}\right)\right\},
$$

it follows by induction from Lemma 2.4 that $m \in f^{-1}(m)$. Thus $m=f(m)$ is a fixed point of $f$.

Lemma 4.4. Let $f: D \rightarrow D$ be a homeomorphism which leaves the root $r$ of $D$ and a point a fixed. Then $f$ maps $M(a)$ homeomorphically onto itself. 
Proof. We see from Lemma 2.5 that $f(M(a)) \subseteq M(a)$, so that $f \mid M(a)$ is an injection. As $f$ is a homeomorphism, its inverse $f^{-1}$ is a homeomorphism too, hence $f^{-1}(M(a)) \subseteq M(a)$ or $M(a) \subseteq f(M(a))$. Therefore $f(M(a))=M(a)$, and $f \mid M(a)$ is a homeomorphism of $M(a)$.

Proof of part (ii) of Theorem 4.1. As a homeomorphism is a monotone map, it only remains to show that the fixed point set of a homeomorphism cannot consist of three points of order three.

Let $a, b$, and $c$ be three distinct fixed points of order three of the homeomorphism $f: D \rightarrow D$. Take $a$ as root. Then

$$
m=\max [L(b) \cap L(c)]
$$

is a fixed point according to Lemma 4.3. So part (ii) of Theorem 4.1 is proved if $m$ is different from $a, b$, and $c$.

Assume now that $m=a$, i.e., that $a$ separates $b$ and $c$. Define

$$
\begin{aligned}
& M_{b}(a)=\{x \mid a \text { separates } b \text { and } x\}, \\
& M_{c}(a)=\{x \mid a \text { separates } c \text { and } x\},
\end{aligned}
$$

(i.e., $M_{b}(a)$ is the set $M(a)$ if $b$ is taken as root, and $M_{c}(a)$ is the set $M(a)$ if $c$ is taken as root). Hence $M_{b}(a)$ and $M_{c}(a)$, and therefore [8, p. 88] the set $Q=M_{b}(a) \cap M_{c}(a)$, are continua. By definition $a \in Q$, but $b \notin Q$ and $c \notin Q$. It follows from Lemma 4.4 (with $b$ resp. $c$ as root) that $f$ induces a homeomorphism of $M_{b}(a)$ and of $M_{c}(a)$, and hence of $Q$. Therefore in this case part (ii) of Theorem 4.1 follows from Theorem 1.1 if we can show that $a$ is of order one in $Q$.

If $a$ is not of order one in $Q$, then $Q \backslash\{a\}$ is not connected. Hence we can select two points $p, q \in Q \backslash\{a\}$ so that $a \in$ arc $p q$ and therefore $a=\max [L(p) \cap L(q)]$. As $q \in M_{b}(a)$, we see that $a$ separates $b$ and $q$. So we have $a \in \operatorname{arc} b q$ and hence $a=\max [L(b) \cap L(q)]$. Similarly $a=\max [L(x) \cap L(y)]$ if $x=b$ or $x=c$, and $y=p$ or $y=q$. This shows that the subdendrite $D^{\prime}=L(b) \cup L(c) \cup L(p) \cup L(q)$ consists of the four arcs [ab], [ac], [ap], and [aq], and that the order of $a$ in $D^{\prime}$ is four. As $a$ is of order three in $D$ this is impossible. So $a$ must be of order one in $Q$, and Theorem 4.1 (ii) holds if $m=a$.

If $m=b$ then $b$ separates $a$ and $c$. Therefore the same argument, but with $b$ and $a$ interchanged, proves Theorem 4.1 (ii) in this case. If $m=c$ we proceed analogously. This concludes the proof of part (ii) of Theorem 4.1, and hence of Theorem 4.1. 
REMARKS. (i) One might ask whether part (ii) of Theorem 4.1 can be extended to monotone maps. That this is not the case is shown by the following example of a monotone surjection of a dendrite which has a fixed point set consisting of three points of order three.

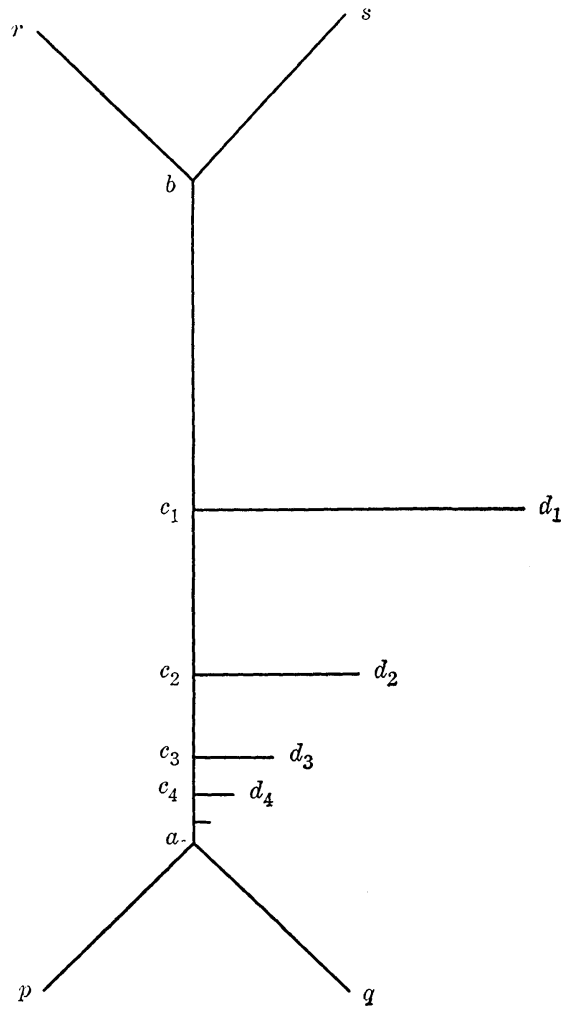

FIGURE 1

Let $D$ be the dendrite illustrated in Figure 1. It is constructed by attaching to the finite dendrite with vertices $a, b, p, q, r$, and $s$ countably many line segments $\left[c_{i} d_{i}\right], i=1,2,3, \cdots$, so that $c_{1}$ is the mid point of $[a b]$, that $c_{i+1}$ is the mid point of $\left[a c_{i}\right]$ for $i=1,2,3, \cdots$, and that the length of $\left[c_{i} d_{i}\right]$ equals the length of $\left[a c_{i}\right]$. Define $f: D \rightarrow D$ first on the vertices of $D$ as follows:

$$
\begin{aligned}
& f(x)=x \text { if } x=a, b \text {, or } c_{1}, \\
& f(p)=q, f(q)=p, f(r)=s, f(s)=r, \\
& f\left(d_{1}\right)=c_{1}, \\
& \left.\begin{array}{l}
f\left(c_{i+1}\right)=c_{i} \\
f\left(d_{i+1}\right)=d_{i}
\end{array}\right\} \text { for } i=1,2,3, \cdots .
\end{aligned}
$$

Now extend $f$ linearly over $D \backslash\left[c_{1} b\right]$, and define it over $\left[c_{1} b\right]$ as a 
monotone map of $\left[c_{1} b\right]$ onto itself with the only fixed points $c_{1}$ and $b$. Then $f: D \rightarrow D$ is monotone and has the fixed points $a, b$, and $c_{1}$, each of order three.

(ii) Tempted by one's habit of mathematical induction one might also ask whether it is possible to prove the nonexistence of a fixed point set under a homeomorphism consisting of $n$ points of order $n$ if $n>3$. That this cannot be done is shown by the next example, in which for any positive integer $n>3$ a dendrite $D_{n}$ and a homeomorphism $f_{n}$ of $D_{n}$ are constructed so that the fixed point set of $f_{n}$ consists of exactly $n$ points of order $n$.

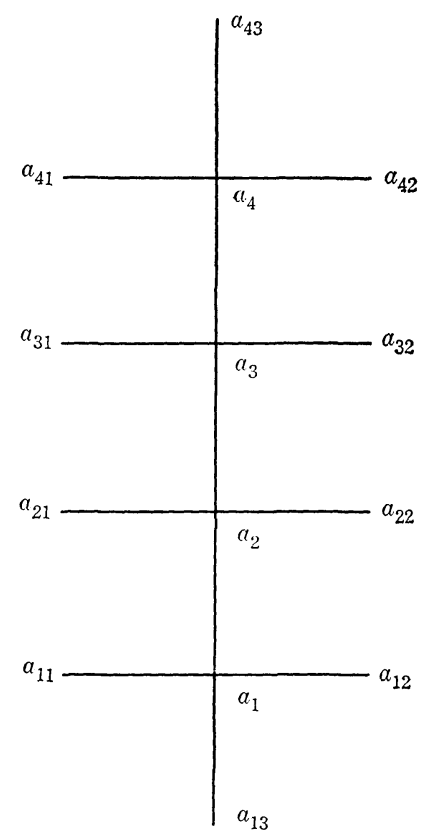

FIGURE 2

Take a chain of $n$ vertices $a_{1}, a_{2}, \cdots, a_{n}$ (see Figure 2 for the case $n=4)$. To both $a_{1}$ and $a_{n}$ attach $n-1$ segments with end points $a_{i j}(i=1$ or $n ; j=1,2, \cdots, n-1)$; to each of $a_{2}, a_{3}, \cdots, a_{n-1}$ attach $n-2$ segments with end points $a_{i j}(i=2,3, \cdots, n-1 ; j=1,2, \cdots, n-2)$. Then $o\left(a_{i}\right)=n$ for $i=1,2, \cdots, n$. Define $f_{n}$ on the vertices of $D_{n}$ by

$$
\begin{aligned}
f_{n}\left(a_{i}\right) & =a_{i}, & i & =1,2, \cdots, n, \\
f_{n}\left(a_{i j}\right) & =a_{i k}, & i & =1,2, \cdots, n,
\end{aligned}
$$

where $j \neq k$ and $f_{n}\left(a_{i j}\right) \neq f\left(a_{i j^{\prime}}\right)$ if $j \neq j^{\prime}$. Extend $f_{n}$ as a homeomorphism with no further fixed points over all edges of $D_{n}$. Then the fixed point set of $f_{n}$ is $\left\{a_{1}, a_{2}, \cdots, a_{n}\right\}$. 
We conclude this paragraph by strengthening Schweigert's theorem in a different direction.

THEOREM 4.5. If a homeomorphism of a dendrite leaves one end point $e$ fixed, then there exists at least one fixed point distinct from $e$ and of order $\neq 2$.

Proof. Take the fixed end point as root $r$ of the dendrite $D$, and let $A$ be the fixed point set of the homeomorphism $f$ of $D$. It follows from Theorem 1.1 that $A \backslash\{r\} \neq \varnothing$, and hence that $a \neq r$ if $a$ is a maximum of $A$. Assume that $o(\alpha)=2$. Then Lemmas 4.2 and 4.4 show that $f$ induces a homeomorphism of $M(a)$ which leaves the end point $a$ of $M(a)$ fixed. Therefore Theorem 1.1 implies the existence of a fixed point of $f$ on $M(a)$ different from $a$, in contradiction to $a=\max A$. So it is necessary that $o(a) \neq 2$.

REMARK. Theorem 4.5 cannot be extended to monotone maps, for we can construct a monotone surjection of a dendrite such that its fixed point set consists of one point of order one and of one point of order two. For this purpose, let $D^{\prime}$ be the subdendrite obtained from the dendrite $D$ in Figure 1 by deleting the end points $p, q, r$, and $s$, and the four edges which have these points as end points. Let $t$ be the mid point of $c_{1}$ and $b$. Define $f$ on the vertices of $D^{\prime}$ as follows:

$$
\left.\begin{array}{rl}
f(a) & =a, f(t)=t, \\
f(b) & =t, f\left(c_{1}\right)=t, \\
f\left(d_{1}\right) & =b, \\
f\left(c_{i+1}\right) & =c_{i} \\
f\left(d_{i+1}\right) & =d_{i}
\end{array}\right\} \text { for } i=1,2,3, \cdots,
$$

and extend it linearly over the edges of $D^{\prime}$. Then $f$ is a monotone map $D^{\prime} \rightarrow D^{\prime}$ with fixed points $a$ of order one and $t$ of order two.

5. Fixed point sets of monotone maps which leave almost all end points fixed. Theorem 1.1 by Schweigert and Ward considers the case where a map is known to leave one end point fixed. The main result of this paragraph, Theorem 5.1, considers a case which is, in a sense, the other extreme: a map is known to leave almost all, or all, of the end points fixed.

THEOREM 5.1. Let $f: D \rightarrow D$ be a monotone surjection of a dendrite $D$ and assume that at most $n$ of the end points of $D$ do not belong to the fixed point set of $f$. Then every point of order $n$ (where 
$n \geqq 3$ ) belongs to the fixed point set of $f$.

We will prove Theorem 5.1 with the help of the following two lemmas.

Lemma 5.2. If $a \in D$, then every component of $D \backslash\{a\}$ contains either the root or an end point.

Proof. Let $K$ be a component of $D \backslash\{a\}$, and let $r$ be the root of $D$. We can assume that $r \notin K$. Take an arbitrary $x \in K$. Then $x \neq r$ and $x \neq a$. For any $y \in M(x) \backslash\{x\}$ we have $a \notin[x y]$, as $x<a \leqq y$ would imply $[r x] \subset D \backslash\{a\}$ and hence $x$ would be contained in the same component of $D \backslash\{a\}$ as $r$. Therefore $M(x) \subset K$. But $M(x)$ is closed in $D$ and hence has a maximum. According to Lemma 2.2 this is an end point, as a maximum of $M(x)$ is clearly a maximum of $D$.

Lemma 5.3. Let $f: D \rightarrow D$ be a monotone surjection which leaves the root of $D$ fixed. Then the counterimage of any maximum of $D$ contains a maximum.

Proof. Let $m$ be a maximum. As $f$ is surjective, there exists an $x \in D$ with $f(x)=m$. As $f$ is isotone (Lemma 2.5), we have $f(M(x))=m$, and as $M(x)$ contains a maximum, so does $f^{-1}(m)$.

Proof of Theorem 5.1. Let $a \in D$ be a point of order $n$ (with $n \geqq 3$ ). If $D$ has only $n$ end points, then it is a finite dendrite of the form $\cup_{i=1}^{n}\left[a e_{i}\right]$, where $\left[a e_{i}\right]$ are arcs. Hence $a$ is fixed. (This can easily be seen directly; it also follows from the arguments used in the proof of Theorem 6.1 below.)

If $D$ has more than $n$ end points, then at least one of them belongs to the fixed point set of $f$; take it as the root $r$ of $D$. According to Lemma 5.2 we can select in each of the components of $D \backslash\{a\}$ which do not contain $r$ an end point, thus obtaining at least $n-1$. Choose them as fixed points if possible, and then select from this set exactly $n-1$ end points, again including as many fixed points as possible. We now continue with the proof by investigating three possible cases.

Case 1. At least two of the selected end points, say $e^{\prime}$ and $e^{\prime \prime}$, are fixed points. Consider $m=\max \left[L\left(e^{\prime}\right) \cap L\left(e^{\prime \prime}\right)\right]$, which is fixed in consequence of Lemma 4.3. If $a \neq m$, then arc $e^{\prime} e^{\prime \prime} \subset D \backslash\{a\}$ by Lemma 2.3 , in contradiction to the selection of $e^{\prime}$ and $e^{\prime \prime}$ in different components of $D \backslash\{a\}$. Hence $a=m$ is fixed. 
Case 2. Only one of the selected $n-1$ end points, say $e$, is a fixed point. Let $e_{1}, e_{2}, \cdots, e_{n-2}$ (where $n-2 \geqq 1$ ) be the other selected end points; they are not fixed. If one or two more nonfixed end points of $D$ exist, call them $e_{n-1}$ and $e_{n}$. Otherwise put $e_{n}$ or both of $e_{n-1}$ and $e_{n}$ equal to $e$. Define

$$
m=\max \left[L(e) \cap \bigcap_{i=1}^{n} \mathrm{~L}\left(e_{i}\right)\right] .
$$

It follows from Lemmas 2.2 and 5.3 that the set $\{e\} \cup\left\{e_{i} \mid i=1,2, \cdots, n\right\}$ is permuted by $f$. Hence Lemma 4.3 shows that $m$ is a fixed point. As $e$ and $e_{i}(i=1,2, \cdots, n-2)$ are in different components of $D \backslash\{a\}$, we see that $a=\max \left[L(e) \cap L\left(e_{i}\right)\right]$ and therefore

$$
a=\max \left[L(e) \cap \bigcap_{i=1}^{n-2} L\left(e_{i}\right)\right] .
$$

This implies $m \leqq a$.

If $m=a$, then $a$ is fixed. If $m<a$, then at least one of $e_{n-1}$ or $e_{n}$ is $\ngtr a$. Without loss of generality we can assume that $e_{n-1} \ngtr a$ and that $m=\max \left[L(e) \cap L\left(e_{n-1}\right)\right] . \quad$ As $f$ is isotone, we have $f([m e])=[m e]$, hence there exists an $\mathrm{x}$ with $m<x<e$ and $f(x)=a$. If $f\left(e_{n-1}\right)>a$, then there must also exist a point $y$ with $m<y<e_{n-1}$ and $f(y)=a$. As $f^{-1}(a)$ is connected, it must contain

$$
\max [L(x) \cap L(y)]=\max \left[L(e) \cap L\left(e_{n-1}\right)\right]=m
$$

by Lemma 2.4. But $f(m)=m<a$. Therefore $f\left(e_{n-1}\right) \ngtr a$, and thus $f\left(e_{n-1}\right)=e_{n}$ and $e_{n} \ngtr a$.

Assume now that $f\left(e_{n}\right)>a$. Then there exists a point $z$ with $m<z<e_{n}$ and $f(z)=a$, and $f^{-1}(a)$ contains

$$
k=\max [L(x) \cap L(z)]=\max \left[L(e) \cap L\left(e_{n}\right)\right] .
$$

As $e_{n}>a$, we have $m \leqq k<a$. But as $a \in[m e]$, we see that $m \leqq f(a) \leqq e$, and as $a \in\left[m e_{i}\right]$ for some $e_{i}$ with $i \leqq n-2$ and $f\left(e_{i}\right)=e_{n-1}$, we see that $m \leqq f(a) \leqq e_{n-1}$. As $m=\max \left[L(e) \cap L\left(e_{n-1}\right)\right]$, this implies $f(a)=m$, and therefore $f([m a])=m$ in contradiction to $f(k)=a$. So it is necessary that $f\left(e_{n}\right) \ngtr a$, i.e., $f\left(e_{n}\right)=e_{n-1}$. We can now apply Lemma 4.3 to the set $\{e\} \cup\left\{e_{i} \mid i=1,2, \cdots, n-2\right\}$ to see that $a$ is fixed.

Case 3. None of the selected end points is a fixed point. Denote these end points by $e_{1}, e_{2}, \cdots, e_{n-1}$ (where $n-1 \geqq 2$ ). If one other nonfixed end point exists, call it $e_{n}$, otherwise put $e_{n}=e_{1}$. As in case 2 we see that 


$$
m=\max \left[\bigcap_{i=1}^{n} L\left(e_{i}\right)\right]
$$

is a fixed point, that

$$
\alpha=\max \left[\bigcap_{i=1}^{n-1} L\left(e_{i}\right)\right]
$$

and hence that $m \leqq a$. If $m=a$, then $a$ is fixed. If $m<a$, then $e_{n} \ngtr a$. Choose $e_{i}(i \leqq n-1)$ such that $f\left(e_{i}\right) \neq e_{n}$, i.e., $f\left(e_{i}\right)>a$. Then $a \in f\left(\left[m e_{i}\right]\right)$, hence there exists an $x$ with $m<x<e_{i}$ and $f(x)=a$. But also $a \in f\left(\left[m e_{n}\right]\right)$, therefore there exists $y$ with $m<y<e_{n}$ and $f(y)=a$. Thus the connected set $f^{-1}(a)$ must contain

$$
\max [L(x) \cap L(y)]=\max \left[L\left(e_{i}\right) \cap L\left(e_{n}\right)\right]=m
$$

by Lemma 2.4, so that $f(m)=a$. But $f(m)=m$, so $m<a$ is impossible.

This completes the proof of Theorem 5.1.

Putting $n=3$ in Theorem 5.1 we obtain the following special case.

CoROLlary 5.4. Let $f: D \rightarrow D$ be a monotone surjection of $a$ dendrite $D$ which leaves at most three of the end points not fixed. Then $f$ leaves all branch points fixed.

We conclude this paragraph by formulating one further consequence of Theorem 5.1, which is a complement to the theorem by Schweigert and Ward.

CoRollary 5.5. Let $f: D \rightarrow D$ be a monotone surjection of a dendrite $D$ which leaves at most one of the end points not fixed. Then $f$ leaves all end points and all branch points fixed.

Proof. Assume that we know that $f$ leaves all end points fixed with the possible exception of one end point, say $e$. Take any of the fixed end points as root $r$. It follows from Lemmas 2.2 and 5.3 that $f^{-1}(e)$ contains an end point which must of necessity be $e$. So $f(e)=e$ is fixed. That all branch points of $D$ are fixed follows now from Corollary 5.4.

REMARK. It is not possible to strengthen Theorem 5.1 to include the points with order $n-1$. To see this, consider the finite subdendrite $D^{\prime \prime}$ of Figure 1 with vertices $a, b, p, q, r$, and $s$, define $f$ on 
the vertices of $D^{\prime \prime}$ by $f(a)=b, f(b)=a, f(p)=r, f(q)=s, f(r)=p$, $f(s)=q$, and extend it linearly over the five edges of $D^{\prime \prime}$. Then $f$ leaves none of the four end points of $D^{\prime \prime}$ fixed. Take $n=4$ in Theorem 5.1, and check that the two branch points $a$ and $b$ of order $n-1=3$ are not fixed.

6. Fixed point sets of monotone maps on finite dendrites. In $\S 4$ we found it necessary to distinguish between fixed point sets of monotone maps and fixed point sets of homeomorphisms on dendrites. We will show now that this distinction is superfluous in the case of finite dendrites, i.e., dendrites with finitely many vertices.

THEOREM 6.1. A subset of a finite dendrite $D$ can be the fixed point set of a homeomorphism of $D$ if and only if it can be the fixed point set of a monotone surjection of $D$.

Proof. It is only necessary to show that a subset $A \subset D$ which is the fixed point set of a monotone surjection $f: D \rightarrow D$ can be the fixed point set of a homeomorphism of $D$.

$A$ is nonempty; select a root $r$ of $D$ with $r \in A$. Take the branch points and end points of $D$, as well as $r$ if not yet included, as the set $V$ of vertices of a simplicial complex $K$ which is a triangulation of $D$. We first show that $f \mid V$ determines a simplicial map $\varphi: K \rightarrow K$ (i.e., $f \mid V$ is a function of the vertices of $K$ onto themselves such that adjoining vertices are mapped onto adjoining vertices).

As $D$ is finite, Lemmas 2.2 and 5.3 imply that the image under $f$ of an end point is an end point. Similarly it follows that the image of a branch point is a branch point if we can show that the counterimage of every branch point contains a branch point. Assume by way of contradiction that $b \in D$ is a branch point such that $o(x)=2$ for all $x \in f^{-1}(b)$. As $f^{-1}(b)$ is closed and connected, it must be of the form $[m n]$, where $m \leqq n$ and $[m n]$ is contained in an edge of $D$. As $f$ is isotone (see Lemma 2.5), we have $f(M(n) \backslash\{n\}) \subseteq M(b) \backslash\{b\}$. As $o(n)=2$, the set $M(n) \backslash\{n\}$ is connected, hence $f(M(n) \backslash\{n\})$ is connected. But $o(b)>2$, therefore $M(b) \backslash\{b\}$ is not connected, and thus $f(M(n) \backslash\{n\}) \neq M(b) \backslash\{b\}$. Choose $y \in M(b) \backslash\{b\}$ such that

$$
f^{-1}(y) \cap[M(n) \backslash\{n\}]=\varnothing .
$$

As $f$ is surjective, there exists $x \in D$ with $f(x)=y$, and we see that then $x \ngtr n$ and even $x \ngtr m$. As $f$ is isotone, we have $f([r x])=[r y]$, therefore there exists $x^{\prime} \in D$ with $r<x^{\prime}<x$ and $f\left(x^{\prime}\right)=b$. This implies $m \leqq x^{\prime} \leqq n$ and hence $m<x$ in contradiction to $x>m$. So 
it follows that $f^{-1}(b)$ must contain a branch point.

We complete the argument that $f \mid V$ determines a simplicial map by showing that $f$ maps adjoining vertices of $K$ onto adjoining vertices. Let $a$ and $b$ be adjoining vertices; we can assume that $a<b$. Then $f(a)<f(b)$. If there exists a vertex between $f(a)$ and $f(b)$, then there exists a vertex $c$ which is its counterimage, i.e., $f(a)<f(c)<f(b)$. As $f$ is isotone and as there is no vertex between $a$ and $b$, this implies $c \notin L(b) \cup M(b)$. Let $m=\max [L(b) \cap L(c)]$, then $o(m)>2$. As $m>a$ would imply $m \geqq b$ and $c \in M(b)$, it follows that $m \leqq a$. Now $f([a b])=[f(a) f(b)]$, hence there exists an $x$ with $a<x<b$ and $f(x)=f(c)$. The set $f^{-1} f(c)$ is connected and therefore contains $\max [L(x) \cap L(c)]=\max [L(b) \cap L(c)]=m$, so that $f(m)=f(c)$. As $f$ determines a bijective transformation of $V$, we must have $m=c$. But this would imply $c \leqq a$ in contradiction to $c \notin L(b)$. So the vertex $f(c)$ cannot exist, and $f \mid V$ determines a simplicial map $\varphi: K \rightarrow K$.

As the image of an edge $[a b]$ under a monotone map $f$ must be the edge $[f(a) f(b)]$, it is now easy to check that the fixed point set $A$ of $f$ must be of the following form:

(1) $a \in A$ for every $a \in V$ with $\varphi(a)=a$;

(2) $A \cap(a b)$ is an arbitrary (possibly empty) closed set for every edge [ab] of $D$ with $\varphi(a)=a$ and $\varphi(b)=b$;

(3) $A \cap(a b)=\varnothing$ for all other edges.

But we can construct a homeomorphism of $D$ with the same images of the vertices as $f$ and with this set $A$ as fixed point set. Therefore Theorem 6.1 holds.

Using a theorem by Whyburn [8, p. 182 Theorem 1.1] we can extend Theorem 6.1 to open maps if $D$ is finite and not an interval, for a study of the proof of Whyburn's theorem shows that in this case $f \mid V$ again determines a simplicial map. Hence we have

THEOREM 6.2. If the finite dendrite $D$ is not an interval, then a subset of $D$ can be the fixed point set of a homeomorphism of $D$ if and only if it can be the fixed point set of an open surjection of $D$.

The case where $D$ is an interval has to be excluded, as e.g., the subset $\{1 / 3,2 / 3\}$ of the unit interval [01] can be the fixed point set of an open surjection but not of a homeomorphism. It would be interesting to know whether any or all of the results of $\S 4$ and $\S 5$ generalize to open maps. The method of proof will have to be different, though, as an open map of a dendrite need not be isotone. 


\section{REFERENCES}

1. R. H. Bing, Partitioning continuous curves, Bull. Amer. Math. Soc. 58 (1952), 536-556.

2. R. L. Plunkett, A fixed point theorem for continuous multi-valued transformations, Bull. Amer. Math. Soc. 7 (1956), 160-163.

3. H. Robbin, Some complements to Brouwer's fixed point theorem, Israel J. Math. 5 (1967), 225-226.

4. H. Schirmer, On fixed point sets of homeomorphisms of the n-ball, Israel J. Math. 7 (1969), 46-50.

5. G. E. Schweigert, Fixed elements and periodic types for homeomorphisms on s.l.c. continua, Amer. J. Math. 66 (1944), 229-244.

6. L. E. Ward, Jr., Partially ordered topological spaces, Proc. Amer. Math. Soc. 5 (1954), 144-161.

7. — A note on dendrites and trees, Proc. Amer. Math. Soc. 5 (1954), 992-994.

8. G. T. Whyburn, Analytic Topology, Providence, R.I., 1942.

Received July 9, 1970. This research was partially supported by the National Research Council of Canada (Grant A7579).

CARLETON UNiversity,

OtTaWa, CANADA 


\title{
PACIFIC JOURNAL OF MATHEMATICS
}

\section{EDITORS}

H. SAMELSON

Stanford University

Stanford, California 94305

C. R. HobвY

University of Washington

Seattle, Washington 98105

\section{J. DugunduI}

Department of Mathematics

University of Southern California

Los Angeles, California 90007

RICHARD ARENS

University of California

Los Angeles, California 90024

\section{ASSOCIATE EDITORS}
E. F. BECKENBACH
B. H. Neumann
F. WOLE
K. Yoshida

\section{SUPPORTING INSTITUTIONS}

\author{
UNIVERSITY OF BRITISH COLUMBIA \\ CALIFORNIA INSTITUTE OF TECHNOLOGY \\ UNIVERSITY OF CALIFORNIA \\ MONTANA STATE UNIVERSITY \\ UNIVERSITY OF NEVADA \\ NEW MEXICO STATE UNIVERSITY \\ OREGON STATE UNIVERSITY \\ UNIVERSITY OF OREGON \\ OSAKA UNIVERSITY \\ UNIVERSITY OF SOUTHERN CALIFORNIA
}

\author{
STANFORD UNIVERSITY \\ UNIVERSITY OF TOKYO \\ UNIVERSITY OF UTAH \\ WASHINGTON STATE UNIVERSITY \\ UNIVERSITY OF WASHINGTON

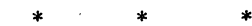 \\ AMERICAN MATHEMATICAL SOCIETY \\ CHEVRON RESEARCH CORPORATION \\ NAVAL WEAPONS CENTER
}

The Supporting Institutions listed above contribute to the cost of publication of this Journal, but they are not owners or publishers and have no responsibility for its content or policies.

Mathematical papers intended for publication in the Pacific Journal of Mathematics should be in typed form or offset-reproduced, (not dittoed), double spaced with large margins. Underline Greek letters in red, German in green, and script in blue. The first paragraph or two must be capable of being used separately as a synopsis of the entire paper. The editorial "we" must not be used in the synopsis, and items of the bibliography should not be cited there unless absolutely necessary, in which case they must be identified by author and Journal, rather than by item number. Manuscripts, in duplicate if possible, may be sent to any one of the four editors. Please classify according to the scheme of Math. Rev. Index to Vol. 39. All other communications to the editors should be addressed to the managing editor, Richard Arens, University of California, Los Angeles, California, 90024.

50 reprints are provided free for each article; additional copies may be obtained at cost in multiples of 50 .

The Pacific Journal of Mathematics is published monthly. Effective with Volume 16 the price per volume (3 numbers) is $\$ 8.00$; single issues, $\$ 3.00$. Special price for current issues to individual faculty members of supporting institutions and to individual members of the American Mathematical Society: $\$ 4.00$ per volume; single issues $\$ 1.50$. Back numbers are available.

Subscriptions, orders for back numbers, and changes of address should be sent to Pacific Journal of Mathematics, 103 Highland Boulevard, Berkeley, California, 94708.

PUBLISHED BY PACIFIC JOURNAL OF MATHEMATICS, A NON-PROFIT CORPORATION

Printed at Kokusai Bunken Insatsusha (International Academic Printing Co., Ltd.), 7-17, Fujimi 2-chome, Chiyoda-ku, Tokyo, Japan. 


\section{Pacific Journal of Mathematics}

\section{Vol. 36, No. $3 \quad$ BadMonth, 1971}

E. M. Alfsen and B. Hirsberg, On dominated extensions in linear subspaces of

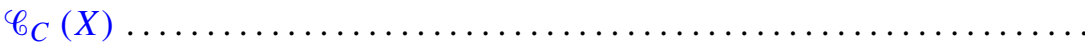

Joby Milo Anthony, Topologies for quotient fields of commutative integral

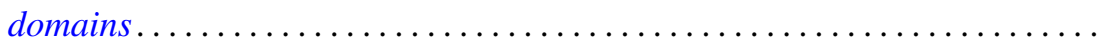

V. Balakrishnan, G. Sankaranarayanan and C. Suyambulingom, Ordered cycle lengths in a random permutation .......................... 603

Victor Allen Belfi, Nontangential homotopy equivalences............... 615

Jane Maxwell Day, Compact semigroups with square roots .............. 623

Norman Henry Eggert, Jr., Quasi regular groups of finite commutative nilpotent algebras .......................................... 631

Paul Erdős and Ernst Gabor Straus, Some number theoretic results ......... 635

George Rudolph Gordh, Jr., Monotone decompositions of irreducible Hausdorff continua .............................................

Darald Joe Hartfiel, The matrix equation $A X B=X \ldots \ldots \ldots \ldots \ldots \ldots . \ldots 69$

James Howard Hedlund, Expansive automorphisms of Banach spaces. II . . . . 671

I. Martin (Irving) Isaacs, The p-parts of character degrees in p-solvable

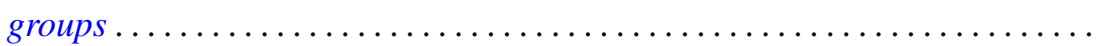

Donald Glen Johnson, Rings of quotients of $\Phi$-algebras ............... 693

Norman Lloyd Johnson, Transition planes constructed from semifield planes....

Anne Bramble Searle Koehler, Quasi-projective and quasi-injective modules.

James J. Kuzmanovich, Completions of Dedekind prime rings as second endomorphism rings...

B. T. Y. Kwee, On generalized translated quasi-Cesàro summability ...

Yves A. Lequain, Differential simplicity and complete integral closure

741

Mordechai Lewin, On nonnegative matrices.

753

Kevin Mor McCrimmon, Speciality of quadratic Jordan algebras ...

Hussain Sayid Nur, Singular perturbations of differential equations in abstract spaces .....................................

D. K. Oates, A non-compact Krein-Milman theorem .

Lavon Barry Page, Operators that commute with a unilateral shift on an invariant subspace...

Helga Schirmer, Properties of fixed point sets on dendrites.

Saharon Shelah, On the number of non-almost isomorphic models of $T$ in a

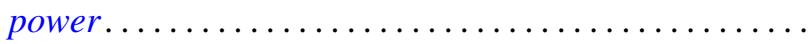

Robert Moffatt Stephenson Jr., Minimal first countable Hausdorff spaces....

Masamichi Takesaki, The quotient algebra of a finite von Neumann algebra 